\title{
Role of Ultrasonography \& Colour Doppler in Scrotal Pathologies
}

Authors

\author{
Drumadala I Gajbhiye ${ }^{1}$, Swenil A Shah ${ }^{2 *}$, Meenakshi I Gajbhiye ${ }^{3}$
}

Dept of Radiology Rajarshi Chhatrapati Shahu Maharaj Govt Medical College \& CPR General Hospital,

Dasara Chowk, Kolhapur 416003

*Corresponding Author

Shah Swenil

\section{Abstract}

Introduction: Ultrasonography (USG) plays a major role in distinguishing intra-testicular from extratesticular abnormalities. The present day diagnostic armamentarium includes gray scale USG, Doppler studies, CT, MRI in addition to radioisotope studies and testicular angiography. While CT and MRI have dominated imaging of other regions of the body, they have certain limitations in evaluation of scrotal diseases in terms of radiation, cost \& availability. USG is exceptionally well suited to study of scrotum and its contents. USG is simple to perform, rapid, non-invasive relatively inexpensive, easily reproducible, widely available and does not involve irradiation of gonads.

Objectives: To evaluate the accuracy of the High Frequency Ultrasound and Colour Doppler in the diagnosis of scrotal pathologies.

Material and Methods: The prospective study was conducted on 200 patients referred for evaluation of scrotal pathology for the period of 2 years from May 2018 to March 2020 from department of surgery and urology.

Results: High frequency grey scale US with Color Doppler sonography accurately differentiates between testicular ischemia / torsion from acute inflammatory diseases in acute painful scrotal conditions. It is also highly sensitive in differentiating intra-testicular from extra testicular origin of scrotal masses. It is highly sensitive in differentiating solid from cystic scrotal masses.

Conclusion: High-frequency ultrasonography and color Doppler sonography has high sensitivity and specificity in diagnosing and evaluating scrotal pathologies. In addition, lack of ionizing radiation, simplicity, wide availability, cost effectiveness and repeatability makes it an extremely valuable modality for scrotal pathologies.

Keywords: Orchitis, Epididymitis, Varicocele, USG, Color Doppler, Scrotum.

\section{Introduction}

Scrotum is a fibro muscular cutaneous bag containing right and left testis, the epididymis and the lower part of the spermatic cord. These structures are affected by a wide variety of pathological conditions which may be congenital, simple inflammatory and neoplastic conditions. ${ }^{1}$
Masses in the scrotum may be grouped into testicular arising from the scrotum and extra testicular arising from other structures in the scrotum. Certain testicular swellings are difficult to diagnose by physical examination alone as well as to differentiate whether arising from testis itself or from extra testicular elements. Physical examination 
alone lacks specificity for evaluating the structures of scrotum and testis. ${ }^{2}$ Hence there is an exceptional need of radiologist in for making diagnosis using imaging modalities. ${ }^{3}$

Ultrasonography has proved to be an exceptional gold standard tool in diagnosis of various scrotal pathologies and conditions. Because of its excellent spatial resolution it has $100 \%$ sensitivity in diagnosis and can differentiate a variety of conditions affecting testis, epidydimis and scrotum with similar clinical manifestations. Gray-scale Ultrasonography (US) in combination with color or power Doppler imaging is a well accepted technique for assessing scrotal lesions and testicular perfusion. ${ }^{4}$ Ultrasonography is exceptionally well suited to study of scrotum and its contents. Sonography is simple to perform, rapid, noninvasive, relatively inexpensive, easily reproducible, and widely available and does not involve irradiation of gonads. Sometimes emergency scrotal Doppler scan are required to diagnose testicular torsion. 4

The present day diagnostic armamentarium for scrotum imaging includes gray scale Ultrasonography, Doppler studies, Computed Tomography \& Magnetic Resonance Imaging, in addition to radioisotope studies and testicular angiography. While CT and MRI have dominated imaging of other regions of the body, they have certain limitations in evaluation of scrotal diseases. CT delivers radiation to gonads, while MRI imaging is costly and not readily available. Thus, this study will be an effort in establishing the role of High frequency Ultrasound as an investigating modality in accurately distinguishing between testicular and extra testicular pathology of scrotum.

\section{Objectives}

1) To evaluate spectrum of ultrasonographic findings in various scrotal pathologies

2) To evaluate value of color doppler sonography in distinguishing and characterizing the blood flow patterns in scrotal pathologies.
3) To assess the role of high frequency real time ultrasonography to distinguish between Testicular and Extra testicular scrotal masses.

\section{Material \& Methods}

Study Design: Prospective Study

Source of Data: The patients will be referred to our department for scrotal ultrasonography and Doppler study by Department of Surgery.

Duration of Study: May 2018- March 2020

Sample size: 200

\section{Inclusion Criteria}

All patients referred from Dept of Surgery for Scrotal Ultrasound and Colour Doppler with pain, swelling or clinically non palpable testis.

\section{Exclusion Criteria}

Patients lost to follow up \& patients with acute scrotal pain with clinical features of torsion testis who were directly taken up for surgery.

\section{Methodology}

Prior to subjecting the patients for ultrasound examination, patient details, detailed clinical history will be obtained along with thorough physical examination. The color Doppler sonography will be routinely performed in all these patients. Subsequently these cases will be followed up and correlated with histopathology report, fine needle aspiration cytology results, surgical findings, response to treatment.

Follow up scans will be done in cases wherever applicable. Abdominal ultrasound scan will be done in conjunction with scrotal scans wherever needed.

Approval of ethical committee will be taken and informed patient consent of all the patients will be taken prior to collecting the data.

Equipment:-High-resolution real time gray scale ultrasonography and Doppler study of scrotum will be carried out using 7.5 to $10 \mathrm{MHz}$ linear transducer; abdominal ultrasonography in required cases will be done using 3.5 to $5.0 \mathrm{MHz}$ convex curved array transducer of Philips HD 11 \& Samsung HS70A Ultrasonography Machines.

Scanning technique: -Scrotal Ultrasound will be performed with the patient in the supine position 
and the scrotum supported by a towel draped over thighs, and the penis is placed on the patient's abdomen and covered with a towel. The testes will be examined in at least two planes, along the long and transverse axes. The size and echogenicity of each testis and the epididymis will be compared with those on the opposite side. Color Doppler and pulsed Doppler parameters will be optimized to display low-flow velocities, to demonstrate blood flow in the testes and surrounding scrotal structures (low wall filter $[100 \mathrm{kHz}]$, low pulse repetition frequency $[1-2 \mathrm{~Hz}]$, and $70 \%-90 \%$ colour gain output settings). Additional techniques such as use of the Valsalva maneuver or upright positioning will be used as needed.

\section{Results}

In the present study conducted, a total of 200 cases were enrolled who fulfilled the inclusion criteria.

No of cases

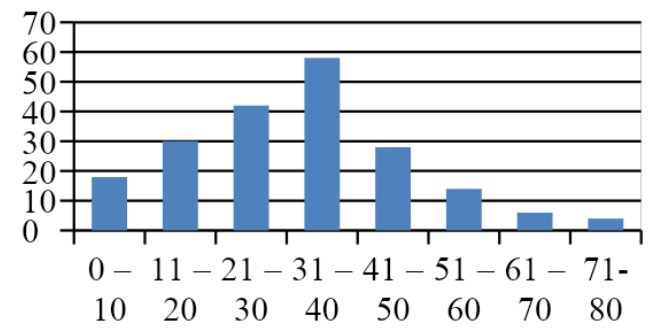

Graph I: Shows the age distributions of cases Highest number of cases presented were in the age group of 31 to 40 years ( 58 cases - 29\%), followed by 21 to 30 years ( 42 cases $-21 \%$ ). The age groups of 21 to 40 years constitute $50 \%$.

Table I: Symptomatology of Patients in the Study

\begin{tabular}{|l|c|}
\hline SYMPTOMS & NO OF CASES \\
\hline Pain and Scrotal Swelling & 68 \\
\hline Pain, Swelling and Fever & 8 \\
\hline Scrotal Swelling & 46 \\
\hline Pain in scrotum & 20 \\
\hline Infertility & 14 \\
\hline Absent testis on palpation & 14 \\
\hline Trauma & 16 \\
\hline Dysuria & 6 \\
\hline Pain abdomen & 6 \\
\hline Erythematous scrotum & 2 \\
\hline
\end{tabular}

Most of the cases clinically presented with combination of multiple symptoms. Commonest clinical presentation was combination of Symptoms like, pain and scrotal swelling, as in 68 cases (34\%), Combination of pain, swelling and fever in 8 cases $(4 \%)$

SPECTRUM OF PATHOLOGIES
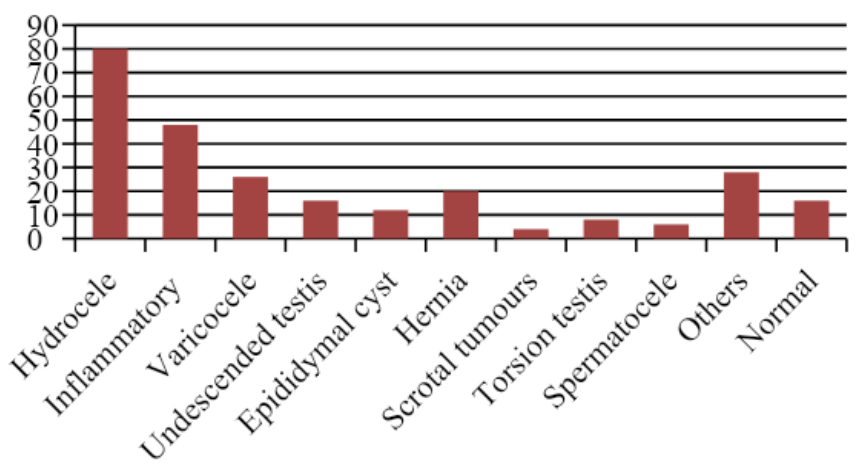

Graph II: Showing Spectrum of Scrotal Pathologies

SIDE DISTRIBUTION

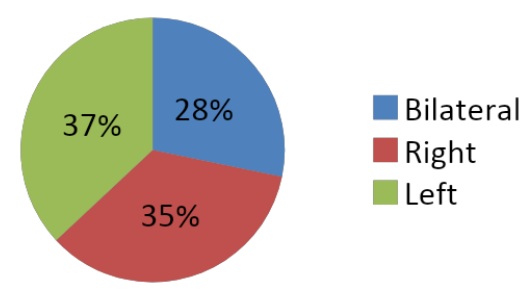

Graph III: Showing Sidewise Distribution

Table II: Distribution of Inflammatory Scrotal Pathologies

\begin{tabular}{|l|c|c|}
\hline Pathology & $\begin{array}{c}\text { No Of } \\
\text { Cases }\end{array}$ & $\begin{array}{c}\text { \% Of } \\
\text { Cases }\end{array}$ \\
\hline Acute Epididymitis & 4 & $8.3 \%$ \\
\hline Acute Epididymo Orchitis & 16 & $33.3 \%$ \\
\hline Acute Orchitis & 5 & $10.4 \%$ \\
\hline Chronic Epididymitis & 4 & $8.3 \%$ \\
\hline Chronic Epididymo Orchitis & 7 & $14.5 \%$ \\
\hline Scrotal Wall Inflammation & 4 & $8.3 \%$ \\
\hline Scrotal Filariasis & 2 & $4.1 \%$ \\
\hline Funiculitis & 3 & $6.2 \%$ \\
\hline Tubercular Epididymo Orchitis & 2 & $4.1 \%$ \\
\hline Fournier's Gangrene & 1 & $2.0 \%$ \\
\hline TOTAL & 48 & \\
\hline
\end{tabular}

In our study, out of 200 cases, 48 cases were detected have inflammatory scrotal pathology on high frequency US and Doppler study. Acute Epididymo orchitis was the commonest inflammatory pathology detected, noted in 16 cases $(33.3 \%)$. Next most frequent inflammatory 
pathology detected was chronic epididymo orchitis, noted in 7 cases $(14.5 \%)$.

\section{VARICOCELE}

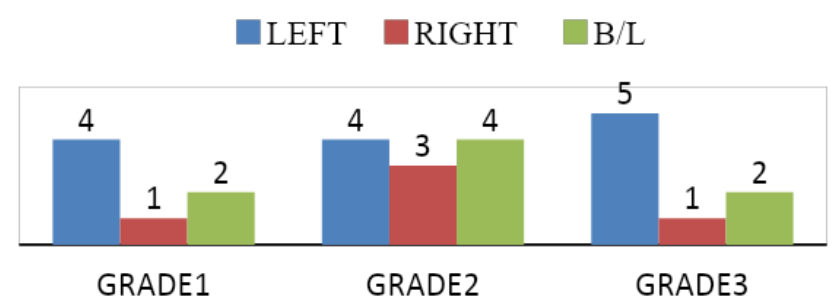

Graph IV: Showing Spectrum of Varicocele

Table III: Non-Neoplastic Swellings of Scrotum

\begin{tabular}{|l|c|c|}
\hline Pathology & $\begin{array}{c}\text { No Of } \\
\text { Cases }\end{array}$ & $\begin{array}{c}\% \text { Of } \\
\text { Cases }\end{array}$ \\
\hline Hydrocele & 80 & $86.9 \%$ \\
\hline Epididymal Cyst & 12 & $13 \%$ \\
\hline Spermatocele & 6 & $7 \%$ \\
\hline Varicocele & 26 & $28 \%$ \\
\hline Testicular Cyst & -- & -- \\
\hline Total & 92 & \\
\hline
\end{tabular}

Among non-neoplastic scrotal swellings, hydrocele is the commonest pathology noted 80 cases (52\%). The incidence of non-neoplastic scrotal swellings is very much high compared to neoplastic swellings. Incidence of extra testicular swellings is more, compared to intra testicular swellings.

Table IV: Spectrum Showing Neoplastic Scrotal Swellings

\begin{tabular}{|l|c|}
\hline TYPES & No of Cases \\
\hline Seminoma & 2 \\
\hline Yolk sac tumour & 1 \\
\hline Teratoma & 1 \\
\hline
\end{tabular}
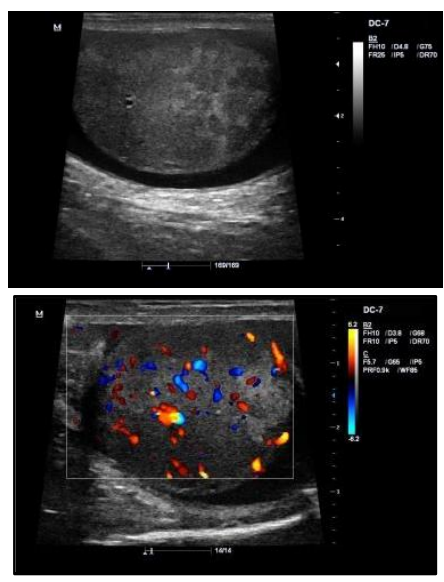
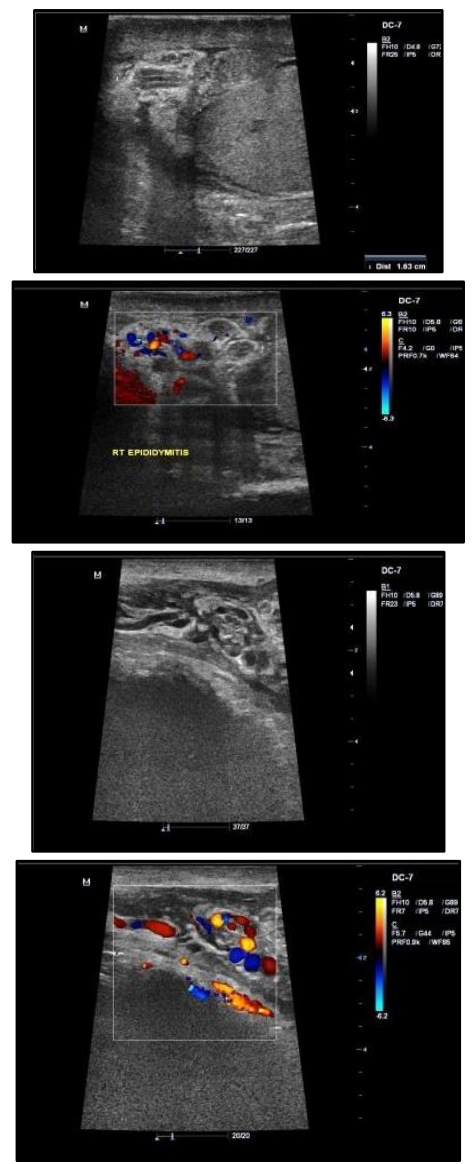

Fig I: Left Sided Acute Epididymo-Orchitis with Acute funiculitis: a)Poorly defined, focal, peripherally situated hypoechoic intratesticular lesions. a), b) The testis is diffusely enlarged and ultrasonic probe tenderness may be present. c) Reduced echogenicity of the epididymis, increase in size of epididymis, secondary hydrocele, scrotal wall thickening .d), e) Thickened echogenic spermatic cord b), d), f) Color Doppler study shows increased blood flow.

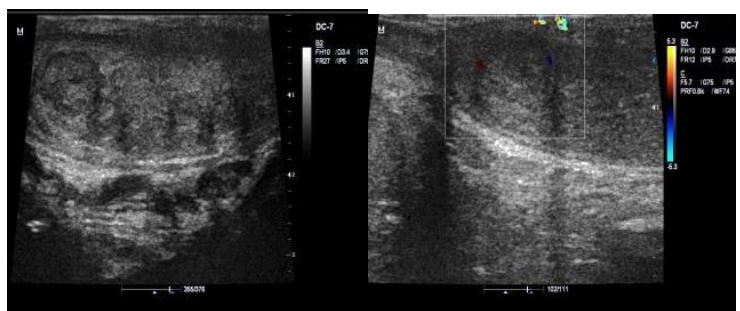

Fig II: Chronic (Tuberculous) Epididymo-orchitis: a), b) On High frequency ultrasound scan, tuberculous testis and epididymis appears as diffusely enlarged heterogeneously hypoechoic, or nodular enlarged heterogeneously hypoechoic lesions. 

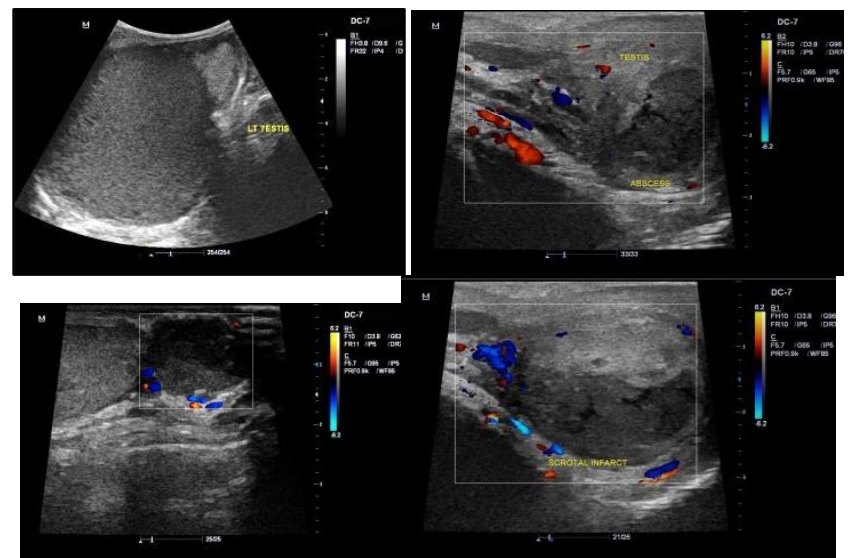

Fig III: Complications of Epididymo-Orchitis: a) Pyocele: It forms due to rupture of a testicular or epididymal abscess into the tunical sac. On scan, pyocele appears as a loculated fluid collection with multiple internal echoes; fluid-fluid and fluid-debris levels may be seen b) Testicular Abscess c)Epididymal Abscess: On High frequency US scan appears as focal fluid collection with low-level echoes. Small abscess may show resolution on follow-up scans after treatment. d) Testicular Segemntal infarct: High frequency US demonstrates enlargement of testis and uniformly hypoechoic texture. Scrotal wall edema and a small reactive hydrocele are usually present.

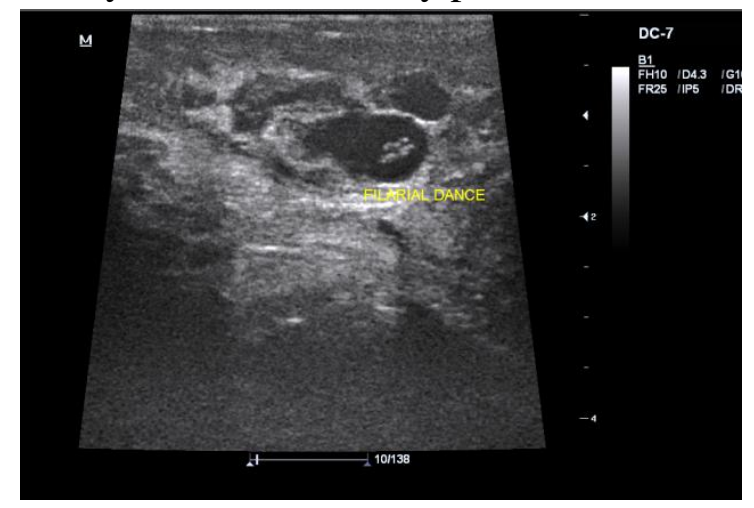

Fig IV: Scrotal Filariasis: Micro-filaria appear as echogenic slender moving structures on real time gray sacle ultrasonography within the spermatic cord and/or epididymii uni or bilaterally within dilated lymphatics which appear as cystic tubular structures.

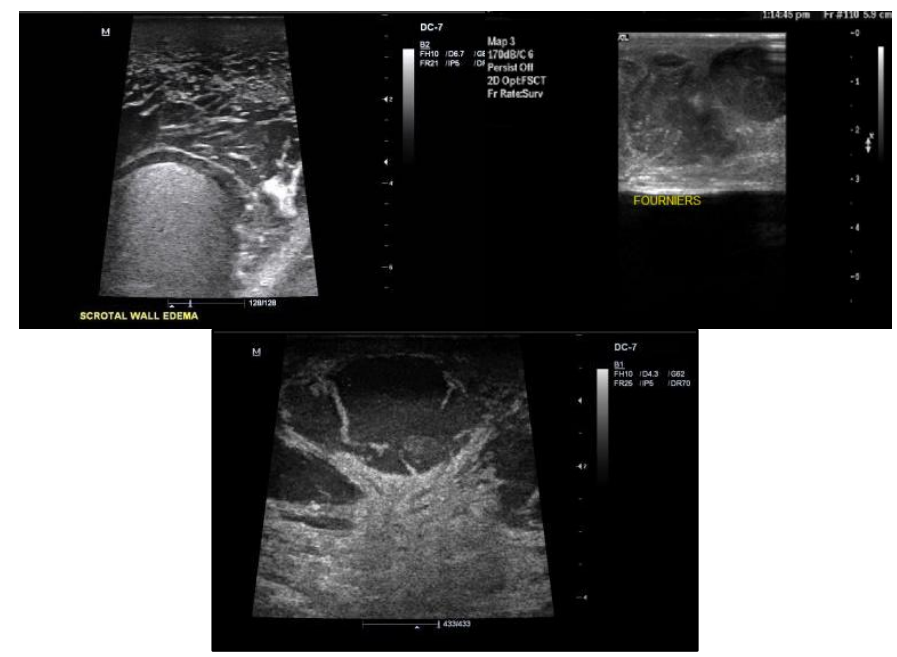

Fig V: Scrotal Wall Pathologies:- a) Scrotal wall edema: Normal scrotal wall thickness is $2-8 \mathrm{~mm}$. Scrotal wall oedema occurs secondary to acute inflammatory pathologies of the scrotum or its contents. On USG, cellulitis of scrotal wall manifests as thickening of scrotal wall, loss of uniform hyper echogenicity of scrotal wall b) Fournier's Gangrene: USG shows scrotal wall thickening, loss of normal hyper echogenicity of the scrotal wall. The hyperechoic band like region is replaced by multiple varying echogenicity. The fluid accumulation occurs in the connective tissue layer between the dartos \& cremasteric fascia and this layer appears hypoechoic on ultrasound. c) Scrotal wall abscess: It manifests as, a focal hypoechoic lesion with low level echoes intralesionally.

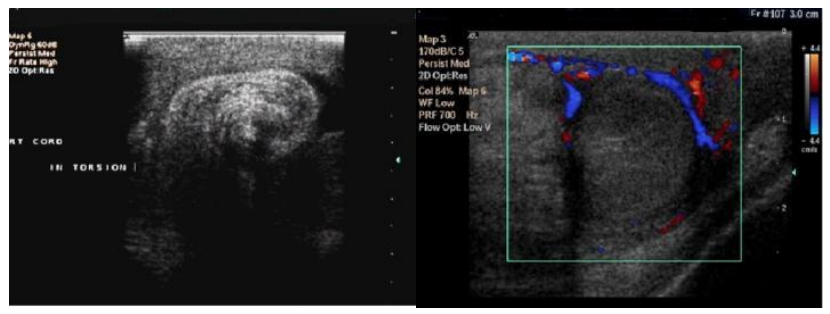

Fig VI: Torsion testis: a) Spermatic cord twisting at the external inguinal orifice called "whirl pool sign" is the most definitive sign of torsion. b) Enlarged testis and heterogeneous echogenicity, absent flow in testis with increased surrounding vascularity. The intrascrotal portion of the cord appears as edematous, round, ovoid or curled echogenic extra testicular mass, with the epididymal head wrapped around it .The definitive diagnosis of complete testicular torsion is made when blood flow is 
visualized on the normal side but is absent on the affected side
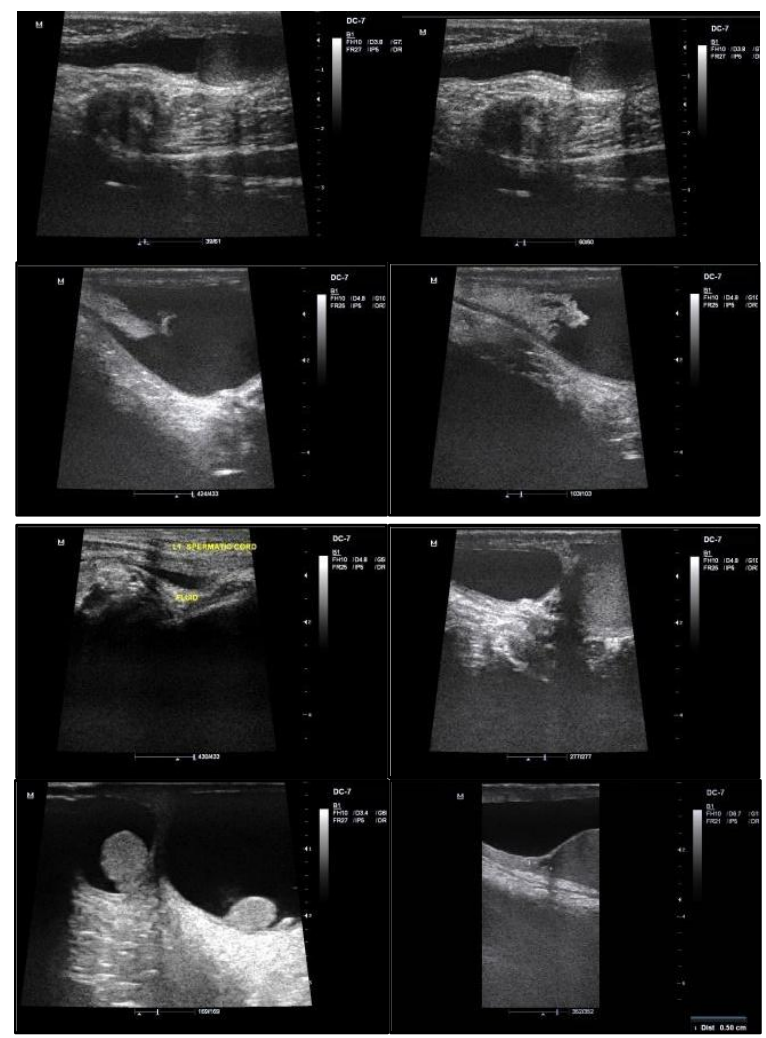

Fig VII: Types of Hydrocele: a), b) Congenital hydrocele, c), d) Congenital Hydrocele with omental herniation, e)Funicular hydrocele, f)Encysted hydrocele of the cord g), h)Primary idiopathic hydrocele.

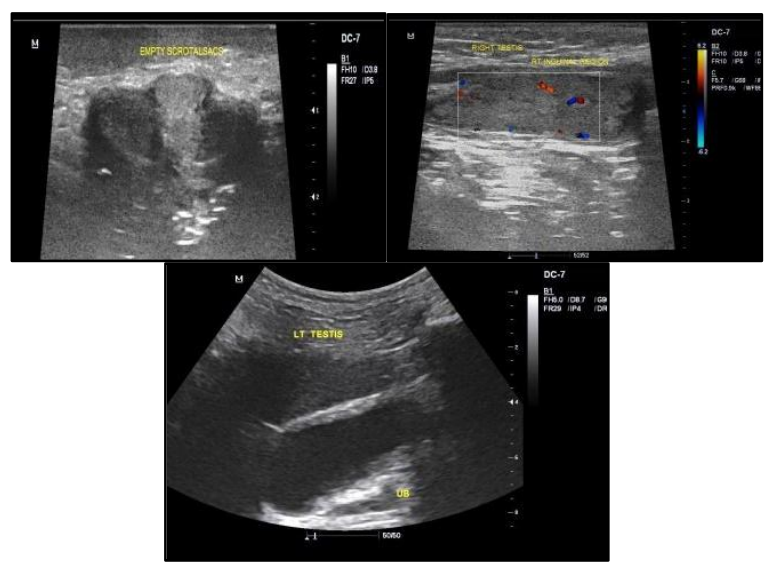

Fig VIII Bilateral Undescended Testis: a) empty scrotal sacs b) right undescended testis in right mid inguinal canal appearing as oval well defined hypo to isoechoic structure showing intra testicular vascularity. c) left undescended testis in left deep inguinal ring anterior and left lateral to urinary bladder with more or less similar echotexture as right testis.

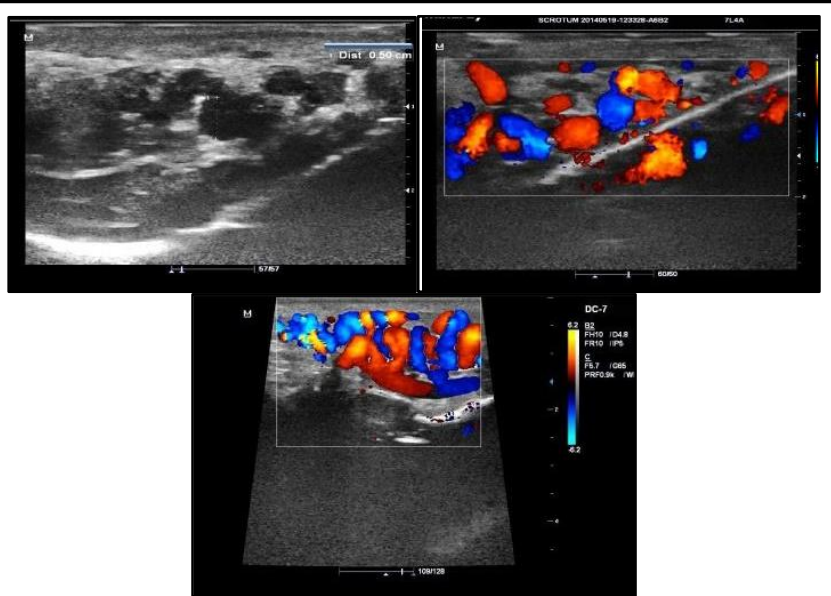

Fig IX: Varicocele: Grade III Varicocele appearing as numerous dilated anechoic tubular / serpigenous structures situated posterior to the testes, in the spermatic cord, in the neck of scrotum and in the inguinal canal. Mean diameter of dominant vein is 5 $\mathrm{mm}$ with more than $1.5 \mathrm{~mm}$ increase on Valsalva maneuver.

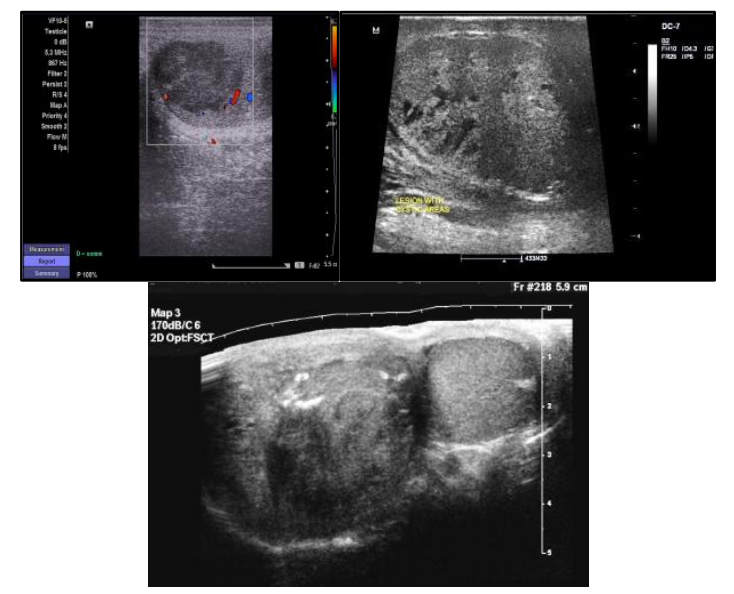

Fig X: Neoplastic Testicular Swellings: a)Seminoma: Enlarged testis showing a lobulated hypoechoic solid mass lesion with minimal to mild internal vascularity. b) Yolk sac tumour appearing as an illdefined heterogeneously hypoechoic lesion within the test is showing cystic areas within. c) Teratoma: Enlarged testis showing markedly heterogenous lesion with multiple cystic areas. Bright echogenic foci with distal acoustic shadowing represents immature bone elements or without distal shadowing representing fibrous connective tissue elements and hair are observed.

\section{Discussion}

The superficial location of the scrotal contents makes them ideally suited for sonographic examination. The development of high frequency, 
real time USG with colour doppler scanners has enhanced the diagnostic accuracy of scrotal sonographic examinations. Scrotal ultrasound has reached a level of maturity that allows the technique to be the first and only imaging examination necessary to evaluate the scrotal contents.

The age distributions of cases in this study are described in table-1 which varied from 2 Years to 73 Years. Highest number of cases presented were in the age group of 31 to 40 years (58 cases $-29 \%$ ), followed by 21 to 30 years (42 cases - 21\%). Commonest clinical presentation was combination of symptoms like, pain and scrotal swelling, as in 68 cases (34\%), combination of pain, swelling and fever in 8 cases (4\%). 14 cases of infertility were referred, which constitute around (7\%). Absent testis was a presenting complaint in 14 cases $(7 \%)$.

Out of 200 cases of study, the pathological process were detected in 184 cases and 16 cases showed normal study. Hydrocele was the most common pathology noted in 40 cases, followed by epididymo orchitis noted in 48 cases, varicoceles were noted in 26 cases, Undescended testis was noted in 16 cases , Inguinal hernia was noted in 20 cases, Epididymal cyst is noted in 12 cases, scrotal neoplastic lesions noted in 4 cases, spermatoceles in 6 cases. Miscellaneous conditions like tortion testis in cases, scrotal filariasis, testicular microlithiasis and calcification in 28 cases. Our study was consistent with study of Donald P Orr et al ${ }^{(5)}$ conducted a prospective study in 200 cases. In our study hydrocele was the most common pathology noted, followed by epididymo-orchitis noted in 48 cases it is comparable for the other studies. Arger et $\mathrm{al}^{(6)}$, in a series of 62 patients, detected inflammatory diseases in 16 cases (26\%), and non-inflammatory swellings in 45 cases. $(67 \%)$. Willscher et $\mathrm{al}^{(7)}$, in a study of 43 pts (86 testes), noted inflammatory diseases in 12 cases, non-inflammatory diseases in 28 cases.

USG is highly sensitive and specific (100\%), in identifying the cause and type of hydrocele and knowing the cause of swelling is either intratesticular or extra testicular compared to physical examination and has a positive predictive value of (100\%). The Chi square statistic is 15.6087 and the $\mathrm{P}$ value is 0.000120 This result is significant at $\mathrm{p}<0.05$.

Horstman, Middleton, and Nelson ${ }^{(8)}$, in their study of 45 patients, found acute epididymitis present in 25 cases $(56 \%)$, acute orchitis in 1 case $(2 \%)$. No case of chronic Epididymo orchitis was reported. Lerner et al, ${ }^{(9)}$ in their limited series of 5 cases of acute inflammatory diseases of scrotum, found acute epididymitis in 3 patients $(60 \%)$, acute Epididymo orchitis in 2 patients (40\%). Farriol et al ${ }^{(10)}$, in their study of 25 cases of acute inflammatory diseases of scrotum using high-resolution grey scale and power Doppler sonographic study, found epididymitis in 11 cases (44\%), Epididymo-orchitis in 10 cases $(40 \%)$, orchitis in 2 cases $(8 \%)$, funiculitis in 2 cases ( $8 \%$ ). In our study of 48 cases of scrotal inflammatory pathologies, we observed 7 cases of complications of acute scrotal pathology, out of which 4 cases are scrotal wall Cellulitis, 1 case of Fournier's gangrene and 2 cases of funiculitis. In Cellulitis of scrotal wall, USG showed loss of normal uniform hypoechoic appearance of scrotal wall, thickening of scrotal wall, presence of normal testis, epididymis and tunical sac. These findings are similar to those of Luker and Siegel ${ }^{(11)}$. On color Doppler sonography, there was evidence of diffuse increase in vascularity in 9 cases, normal vascularity in 2 cases, focal increase in vascularity in 2 cases, findings are in similarity with study KIM S H et $\mathrm{al}^{(12)}$.

Our results indicate that color Doppler sonography is having high sensitivity (100\%), compared to physical examination. These findings were compared to previous similar study by Randall B Meacham et $\mathrm{al}^{(13)} \&$ Petros $\mathrm{J} A$ et $\mathrm{al}^{(14)}$. Color Doppler sonography is having high sensitivity (100\%) and PPV (95\%), compared to physical examination.

In our study 4 scrotal neoplasms diagnosed, of which all of them were intra testicular. Rickfeld and middleton ${ }^{(15)}$ described the features of seminoma on ultrasound as, homogenous, hypoechoic, oval shaped or round, sharp demarcation, possible multifocal involvement. These features were 
correlating with our study. Our study had a sensitivity of $75 \%$ and a positive predictive value of $100 \%$ in evaluation of testicular tumours.

In our study 8 cases of testicular torsion noted, all case presented clinically with acute onset of pain and swelling. Grey scale showed change in axis, with oedema. On colour Doppler showed absent colour flow in all eight cases. Two cases showed peripheral increase in colour flow suggestive of sub acute torsion, which was confirmed surgically as necrosis. One case of torsion presented within 6 hours, was diagnosed early and orchidopexy follow up showed good reperfusion. All cases showed torsion knot on gray scale at the root of scrotum, where epididymis wound around the cord to form torsion knot complex. The color Doppler confirmation showed whirl pool sign appearance of spermatic cord in cross section. Clinically suspected torsion was diagnosed as acute epididymo-orchitis in 20 cases, which avoided unnecessary surgical intervention.

Thus in our study, the overall diagnostic validity of high resolution Ultrasonography and Colour Doppler was high with sensitivity of $95 \%$ and specificity of $80 \%$.

\section{Conclusions}

Gray-scale USG is an accurate, fast and useful imaging modality for the imaging of scrotal lesions. High-frequency ultrasonography enables in clear demonstration of morphological alterations associated with acute scrotal inflammatory diseases, and color Doppler study is highly sensitive in diagnosing acute scrotal pathology. In addition, Color Doppler USG is the modality of choice to accurately differentiate between testicular ischemia and torsion from acute inflammatory diseases in acute painful scrotal conditions and can thus help in avoiding unnecessary surgical explorations. USG is highly sensitive in differentiating solid from cystic scrotal masses. It is highly sensitive in distinguishing scrotal mass as either testicular or extra testicular masses and is clearly superior to clinical diagnosis. Color Doppler enhances the visualization of varicoceles. We conclude that High- frequency ultrasonography and color Doppler sonography is an extremely valuable and highly sensitive tool in evaluation of scrotal and testicular pathologies.

\section{Source of Support/grants: Nil}

\section{References}

1. Adekanmi AJ, Adeniji-Sofoluwe AT, Obajimi G, Okafor E. A 10-Year review of ultrasonographic findings of scrotal diseases in Ibadan, South Western, Nigeria. Afr J Med Health Sci; 2018;17:60-5.

2. Priyan Voltaire R, Ritesh Kondeti. A study on evaluation of scrotal lesions by gray scale and colour doppler ultrasonography at a tertiary care hospital of south India. International Journal of Contemporary Medicine Surgery and Radiology. 2018;3(3):C153-C157. doi: 10.21276/ijcmsr.2018.3.3.34

3. Bhardwaj Patiala: Role of Color Doppler in Scrotal Lesions. Indian J Radiol Imaging. 2009 Aug; 19(3): 187-190. doi: 10.4103/0971-3026.54874: 10.4103/0971-3026.54874.

4. Ibrahim MZ, Tabari AM, Igashi JB, Lawal S, Ahmed M. Scrotal doppler ultrasound evaluation in Zaria, Nigeria. Niger J Basic Clin Sci 2016;13:89-93.

5. Donald Orr, Skonlunick ML : Sonographic evaluation of the abnormal scrotum. Clinical Radiology 31: 109- 113, 1980.

6. Peter $\mathrm{H}$ Arger, et al, Prospective analysis of the value of Scrotal Ultrasound. Radiology 1981; 141: 763-766.

7. Max $\mathrm{K}$ Willscher et al, Scrotal Ultrasonography. The Journal of Urology, 1983; 130:931 - 935.

8. Horstman W G, Scrotal inflammatory disease-color Doppler evaluation, Radiology1991; 179:55-59.

9. Robert M Lerner et al, Color Doppler US in the evaluation of Acute Scrotal Disease, Radiology, 1990; 176:355-358. 
10. Farriol VG, Comella XP, Agromayor EG,Creixams XS, Martinez DL, Torre IB.Gray-scale and power Doppler sonographic appearances of acute inflammatory diseases of the scrotum. $\mathrm{J}$ of Clin Ultrasound 2000;28 : 67-72.

11. Luker, Color Doppler of scrotum in children, AJR 1994; 163:649-655.

12. George R Leopold, et al. High resolution ultrasonography of scrotal pathology Radiology 1979; 131: 719-722.

13. Meacham RB, Townsend RR, Rademacher $\mathrm{D}$, Drose JA. The incidence of varicoceles in the general population when Evaluated by Physical examination, gray scale sonography and color Doppler Sonography, J Urol, 1994; 151: 1535-1538 .

14. Petros JA, Andriole GL, Middleton WD, Picus DA. Correlation of testicular color Doppler US, physical examination and venography in detection of left sided varicocele in men with infertility: J Urol. 1991 Apr;145(4):785-8.

15. Arger PH, Mulhern CB Jr, Coleman BG, Pollack HM, Wein A, Koss J, Arenson R, Banner M.Prospective analysis of the value of Scrotal Ultrasound. Radiology.1981 Dec; 141(3):763-6. 